\title{
Equivalent model of multi-type distributed generators under faults with fast-iterative calculation method based on improved PSO algorithm
}

Puyu Wang ${ }^{1 *}$, Jinyuan Song ${ }^{1}$, Fangyu Liang ${ }^{1}$, Fang Shi ${ }^{2}$, Xiangping Kong ${ }^{3}$, Guangen Xie ${ }^{1}$, Xiao-Ping Zhang ${ }^{4}$ and Xinxin $\mathrm{Gu}^{5}$

\begin{abstract}
There are various types of distributed generators (DGs) with different grid integration strategies. The transient characteristics of the fault currents provided by the DGs are different to those of conventional synchronous generators. In this paper, a distribution network with multi-type DGs is investigated, including consideration of DG low-voltage ride through (LVRT). The fault current characteristics of two typical DGs, i.e. an inverter-interfaced distributed generator (IIDG) and a doubly-fed induction generator (DFIG), are analyzed, considering the specific operation modes. Based on analysis of the fault characteristics, an equivalent model of the multi-type DGs under symmetrical/asymmetrical fault conditions is established. A fast-iterative fault calculation method for enhancing the calculation efficiency while avoiding local convergence is then proposed using an improved particle swarm optimization (PSO) algorithm. A simulation system of the distribution network with multi-type DGs is established in PSCAD/EMTDC. The simulation results validate the high accuracy and calculation efficiency of the proposed calculation method of the fault components. This can assist in the settings of the protection threshold.
\end{abstract}

Keywords: Multi-type distributed generators (DGs), Fault current characteristics, Equivalent model, Fast-iterative calculation method, Improved particle swarm optimization (PSO)

\section{Introduction}

With the rapid development of the modern distribution network and the access of distributed generation, the structure of distribution network is becoming increasingly complex $[1,2]$. New challenges have been raised in the relay protection of a distribution network because of the integration of various types of distributed generators (DGs) and increased penetration of DGs [3, 4]. To ensure safe and stable operation of the modern distribution network and prevent chain losses of DGs under fault

\footnotetext{
*Correspondence: puyu.wang@hotmail.com

${ }^{1}$ School of Automation, Nanjing University of Science \& Technology

(NUST), Nanjing 210094, Jiangsu, China

Full list of author information is available at the end of the article
}

conditions, several requirements have been formulated requiring DGs to have low-voltage ride through (LVRT) capability [5, 6]. During the LVRT process, DG fault current characteristics are significantly different to those of conventional synchronous generators [7]. This makes it difficult to calculate the fault components and satisfy relay protection requirements [8-10]. Hence, it is necessary to investigate the modelling techniques of a DG integrated distribution network and find effective calculation methods of the fault components under fault conditions.

In general, DGs can be divided into two types according to their forms of integration: asynchronous (e.g., doubly-fed induction generator, DFIG) and inverter-based (e.g., photovoltaic, PV). The fault currents provided by the DGs are closely related to the LVRT strategies, forms 
of integration and the protection strategies. Research has been conducted on the analysis of fault current characteristics and establishment of equivalent models of the DGs. For the inverter-based DG, also known as inverterinterfaced distributed generator (IIDG), the nonlinear relationship between the fault current and terminal voltage is analyzed in [11], where the IIDG is represented by an equivalent impedance in series with a constant voltage. However, the corresponding calculation method is not investigated. In [12], a calculation method of a distribution network under faults is investigated and both sub-transient and steady state equivalent models are established. However, the fault current characteristics are not analyzed. In [13], the fault current characteristics of an IIDG in microgrids are studied, though the control strategy in the islanded operation mode in microgrids is different to that in a distribution network, resulting in different fault current characteristics. For asynchronous type DGs, e.g., DFIG, crowbar protection is generally considered and the back-to-back converter is rapidly blocked during most LVRT processes [14, 15]. The fault characteristics of the DFIG under continuous excitation of the rotor-side inverter have also been considered, such that the DFIG can maintain grid connection and provide voltage/reactive support under specific fault conditions $[16,17]$. A transient equivalent model of the DFIG under continuous excitation is explored in [16] and a calculation method of the fault current is proposed with the assumption of no sudden change of the flux linkage. However, the impact of asymmetrical faults is not considered. In [17], an equivalent model of the DFIG with continuous excitation under faults is analyzed, but the reactive support of the DFIG is not investigated. In [18], the dynamic responses of the DFIG active power are analyzed and a practical equivalent method is proposed, but the fault components cannot be directly obtained from the proposed model.

For the analysis of a distribution network with multiple DGs, different types of equivalent models are proposed in [19] according to the fault location, and an iterative calculation method of the fault current is proposed based on a superposition theorem. However, only IIDG type DGs are considered. In [20], new energy sources with rotating characteristics are proposed as equivalent to the conventional synchronous generator model. However, it is only applicable in specific conditions and cannot be used under single-phase to ground fault.

Thus we see that an equivalent model of multi-type DGs considering variable control strategies has not been established. The impact of the LVRT control strategies on fault current characteristics has not been fully analyzed either, and the existing calculation methods are relatively weak and cannot suffice for practical applications.
Therefore, it is necessary to establish an equivalent model of multi-type DGs and develop an effective calculation method for the distribution network with multi-type DGs.

The rest of this paper is organized as follows. The fault current characteristics of the IIDG and DFIG are analyzed in Sect. 2. They are controlled under LVRT requirements and with continuous excitation of the rotor-side inverter, respectively. Based on the analysis of the fault characteristics, an equivalent model of multi-type DGs is then established. In Sect. 3, a fast-iterative calculation method for the distribution network with multi-type DGs, with enhanced calculation efficiency while avoiding local convergence, is proposed based on a particle swarm optimization (PSO) algorithm. A simulation system of the distribution network with multi-type DGs is established in PSCAD/EMTDC in Sect. 4 and the simulation results verify the theoretical analysis and the proposed fast-iterative calculation method.

\section{Equivalent model of multi-type DGs}

\subsection{Fault current characteristics of the IIDG}

The fault current characteristics of an IIDG are closely related to the LVRT strategy. In order to accurately analyze the fault characteristics, a LVRT strategy that meets the requirements should be established at the initial stage. A typical LVRT strategy [21] is depicted in Fig. 1. As shown, when a fault occurs resulting in voltage drop, an IIDG should remain connected to the grid at the initial $0.625 \mathrm{~s}$. After $0.625 \mathrm{~s}$, if the voltage remains lower than the diagonal line, the DG can be disconnected from the grid. In addition, to meet the LVRT requirement, the IIDG needs to adjust the reactive current according to the voltage drop to provide sufficient reactive support [22]:

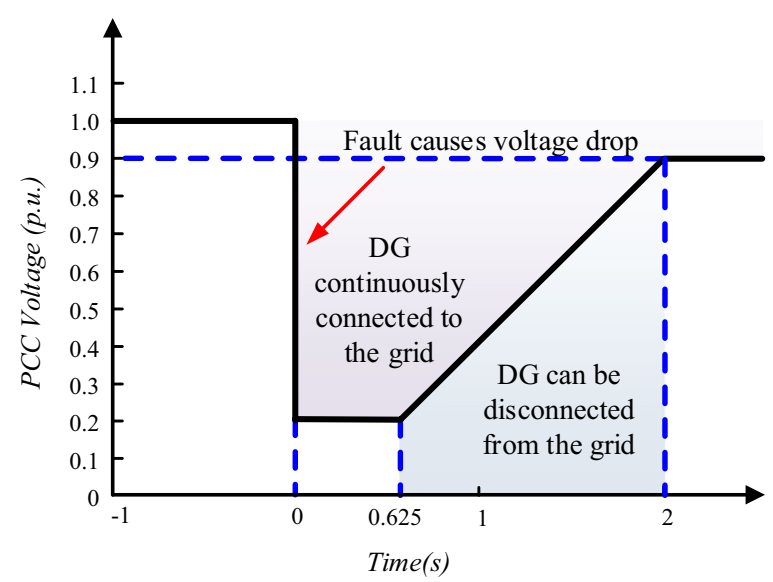

Fig. 1 A typical LVRT strategy 


$$
i_{\text {qref }}= \begin{cases}0 & V_{p c c}>0.9 \\ \min \left\{I_{\max }, k \cdot\left(1-V_{p c c}\right)\right\} & 0.2<V_{p c c}<0.9\end{cases}
$$

where $i_{\text {qref }}$ is the output current reference of the $q$-axis component, $k$ represents the required reactive current coefficient, $V_{p c c}$ is the voltage at the point of common coupling $(P C C)$, and $I_{\text {max }}$ is the maximum current of the inverter and is generally set to $120 \%$ of the rated current.

To ensure the inverter is operated within the maximum current limit, the active current reference is given as:

$$
i_{d r e f}=\min \left\{i_{d 0 r e f}, \sqrt{I_{\max }^{2}-i_{\text {qref }}^{2}}\right\}
$$

where $i_{d r e f}$ is the reference of the $d$-axis active current, $i_{d 0 \text { ref }}$ is the current reference prior to the fault, and $\sqrt{I_{\max }^{2}-i_{\text {qref }}^{2}}$ is the maximum active current allowed considering the inverter current limit. The three-phase fault currents of the IIDG under a symmetrical fault are [23]:

$$
\left\{\begin{array}{l}
i_{a 1}(t)=\sqrt{i_{d 1}^{2}+i_{q 1}^{2}} \cos \left(\omega_{1} t+\theta+\varphi\right) \\
i_{b 1}(t)=\sqrt{i_{d 1}^{2}+i_{q 1}^{2}} \cos \left(\omega_{1} t+\theta-\frac{2}{3} \pi+\varphi\right) \\
i_{c 1}(t)=\sqrt{i_{d 1}^{2}+i_{q 1}^{2}} \cos \left(\omega_{1} t+\theta+\frac{2}{3} \pi+\varphi\right)
\end{array}\right.
$$

where $i_{a 1}, i_{b 1}$ and $i_{c 1}$ represent the positive-sequence currents of Phase $A, B$ and $C$, respectively. $i_{d 1}$ and $i_{q 1}$ are the active and reactive current references after the fault, $\theta$ is the initial current angle of Phase $A$, and $\phi$ is the current angle after the fault, i.e. $\phi=\arctan \left(i_{q 1} / i_{d 1}\right)$.

The inertia of grid-integrated power-electronics-based units varies based on their size and control. The typical inertia time constant of an IIDG is relatively small, and is approximately $10 \mathrm{~ms}$. As a result, the transient components of an IIDG after faults can be ignored and the steadystate fault component is the main factor that influences the protection threshold setting. It can be seen from (3) that the amplitude and phase of the IIDG output currents have changed and the specific changing ranges are related to the operating condition before the fault and the voltage drop after the fault. For asymmetrical faults, the amplitude and phase of the positive sequence voltage can be locked rapidly and accurately by the phase-locked loop [24]. Reactive and active current references are obtained according to (1) and (2), and the output currents can rapidly track their references through PI regulation of the inner current loop. Under asymmetrical faults, the IIDG output current is the same as that under symmetrical faults shown in (3), indicating that only positive sequence currents are generated under both symmetrical and asymmetrical faults. This is different to conventional synchronous generators. Hence, an IIDG can be equivalent to a positive-sequence current source, whose magnitude depends on the operating condition prior to the fault and the voltage drop at the $P C C$ after the fault.

\subsection{Fault current characteristics of the DFIG}

According to the grid integration requirements [25], a DFIG is required to provide reactive power during the fault period to support the distribution network. Under asymmetrical faults, unbalanced voltages have negative impact on the DFIG, e.g., increased losses on the stator and rotor windings, excessive heat, and emergence of pulsating electromagnetic torque. To ensure safe operation, a control strategy for balancing the stator current, which eliminates the side effects of the negative-sequence components, is applied in this paper. Thus, the rotor-side negative-sequence current can be reduced, and the second harmonic frequency components of the stator active power and electromagnetic torque can be suppressed [26]. The LVRT process of the DFIG under the control strategy is analyzed as follows.

The stator side applies the generator convention for derivation. Considering the positive and negative $d q$ rotating coordinates, the DFIG stator voltage and flux linkage can be derived as [27]:

$$
\begin{gathered}
\left\{\begin{array}{l}
u_{s d}^{+}=-R_{s} i_{s d}^{+}-\omega_{1} \psi_{s q}^{+}+\frac{d \psi_{s d}^{+}}{d t} \\
u_{s q}^{+}=-R_{s} i_{s q}^{+}+\omega_{1} \psi_{s d}^{+}+\frac{d \psi_{s q}^{+}}{d t} \\
u_{s d}^{-}=-R_{s} i_{s d}^{-}-\omega_{2} \psi_{s q}^{-}+\frac{d \psi_{s d}^{-}}{d t} \\
u_{s q}^{-}=-R_{s} i_{s q}^{-}+\omega_{2} \psi_{s d}^{-}+\frac{d \psi_{s q}^{-}}{d t}
\end{array}\right. \\
\left\{\begin{array}{l}
\psi_{s d}^{+}=-L_{s} i_{s d}^{+}+L_{m} i_{r d}^{+} \\
\psi_{s q}^{+}=-L_{s} i_{s q}^{+}+L_{m} i_{r q}^{+} \\
\psi_{s d}^{-}=-L_{s} i_{s d}^{-}+L_{m} i_{r d}^{-} \\
\psi_{s q}^{-}=-L_{s} i_{s q}^{-}+L_{m} i_{r q}^{-}
\end{array}\right.
\end{gathered}
$$

where the superscripts ' + ' and '-' indicate a synchronous rotating $d q$ coordinate under forward/positive and reverse/negative rotations, respectively. The subscripts $s$ and $r$ represent the respective stator and rotor components, while $\omega_{1}$ and $\omega_{2}$ represent the respective positive and negative $d q$ angular velocities, i.e. $\omega_{2}=-\omega_{1}$. The stator voltage is set as the $d$-axis vector reference, and thus:

$$
\left\{\begin{array}{l}
u_{s d}^{+}=u_{s(1)} \\
u_{s q}^{+}=0 \\
u_{s d}^{-}=u_{s(2)} \\
u_{s q}^{-}=0
\end{array}\right.
$$

where $u_{s(1)}$ and $u_{s(2)}$ represent the positive and negative sequence stator voltage components, respectively. During the fault period, a typical approach is to block the outer power control loop, so only the impact of the inner current control loop needs to be considered. In addition, since the inertia time constant of the rotor is large, the change of the rotor speed is much slower than those of 
the electrical components. Hence, the rotor speed can be considered to be constant in the fault analysis.

Under continuous excitation control, the DFIG fault current only contains the fundamental component and an attenuated DC component. In the short-circuit calculation, the fundamental frequency component is predominantly considered and the $d$ and $q$-axis components of the stator flux linkage generally remain unchanged, i.e. $\frac{d \psi_{s d}^{+}}{d t}=\frac{d \psi_{s q}^{+}}{d t}=0$. Thus, the DFIG fault current calculation can be simplified to the calculation of the steady-state fundamental frequency component. Combining (4) and (5) and ignoring the stator resistance yield:

$$
\left\{\begin{array}{l}
u_{s(1)}=\omega_{1} L_{s} i_{s q}^{+}-\omega_{1} L_{m} i_{r q}^{+} \\
0=-\omega_{1} L_{s} i_{s d}^{+}+\omega_{1} L_{m} i_{r d}^{+}
\end{array}\right.
$$

Since the reactive power is regulated based on the voltage drop, the stator reactive current reference can be obtained as:

$$
i_{\text {sqref }}^{+}= \begin{cases}0 & V_{p c c}>0.9 \\ 1.5 \cdot\left(1-V_{p c c}\right) & 0.2<V_{p c c}<0.9\end{cases}
$$

By substituting (8) into (7), the rotor reactive current can be derived as:

$$
i_{r q r e f}^{+}=\frac{L_{s} i_{s q r e f}^{+}}{L_{m}}-\frac{u_{s(1)}}{\omega_{1} L_{m}}
$$

When an asymmetrical fault occurs, the DFIG fault current is composed of both positive and negative sequence components. In order to mitigate the negative sequence component, which may lead to unbalanced heating of the stator winding, a control strategy is applied to eliminate the negative sequence components of the stator current by forcing their references to 0 , i.e.:

$$
\left\{\begin{array}{l}
i_{\text {sdref }}^{-}=0 \\
i_{\text {sqref }}^{-}=0
\end{array}\right.
$$

Under asymmetrical fault conditions, a double frequency $\left(2 \omega_{1}\right)$ component will be generated by the negative sequence component in the forward rotating $d q$ coordinate. This double frequency component can be filtered by a notch filter, represented by [27]:

$$
N(s)=\frac{s^{2}+\omega_{n}^{2}}{s^{2}+2 \omega_{c} s+\omega_{n}^{2}}
$$

where $\omega_{n}$ represents the notch frequency and is $2 \omega_{1}$ in this paper, and $\omega_{c}$ represents the cut-off frequency. A notch filter is added in the phase-locked loop to extract and filter the positive and negative sequence components. Similar to the positive sequence stator flux linkage, there is $\frac{d \psi_{s d}^{+}}{d t}=\frac{d \psi_{s q}^{s}}{d t}=0$ under the steady-state in the fault period. Thus, rotor current references can be obtained in the negative $d q$ coordinate by considering (4) $-(6)$ and (10), as:

$$
\left\{\begin{array}{l}
i_{\text {rdief }}^{-}=0 \\
i_{\text {rqref }}^{-}=u_{s(2)} / \omega_{1} L_{m}
\end{array}\right.
$$

Because of the small capacity of the rotor inverter, the overall rotor current must be limited. Hence, the rotor active current reference is limited to the minimum value of the active current reference prior to the fault $i_{r d 0 r e f}$ and the maximum active current allowed under the current limit, as:

$$
i_{\text {rdref }}^{+}=\min \left\{i_{\text {rdoref }}, \sqrt{I_{\text {rmax }}^{2}-i_{\text {rqref }}^{+2}-i_{\text {rqref }}^{-2}}\right\}
$$

where $I_{\text {rmax }}$ represents the maximum current allowed by the rotor side inverter and is generally set to $120 \%$ of the rated current. According to (4)-(13), the DFIG stator currents under symmetrical and asymmetrical faults are:

$$
\left\{\begin{array}{l}
i_{\text {sdref }}^{+}=\frac{L_{m}}{L_{s}} i_{\text {rdref }}^{+} \\
i_{\text {sqref }}^{+}=1.5 \cdot\left(0.9-V_{p c c}\right) \\
i_{\text {sdref }}^{-}=0 \\
i_{\text {sqref }}^{-}=0
\end{array}\right.
$$

The rotor side inner current loop is controlled by PI feedforward control to track the current references, which can be designed with Type I/Type II controller to ensure:

$$
\left\{\begin{array}{l}
i_{s d}=i_{\text {sdref }}^{+} \\
i_{s q}=i_{\text {sqref }}^{+}
\end{array}\right.
$$

where $i_{s d}$ and $i_{s q}$ represent the $d$-axis and $q$-axis components of the DFIG stator current, respectively. The grid side converter is generally controlled with unit power factor operation to reduce the reactive current impact on the distribution network. In addition, the grid side converter eliminates the negative-sequence current under asymmetrical faults, so the output $d$-axis and $q$-axis current components $i_{g d}$ and $i_{g q}$ are:

$$
\left\{\begin{array}{l}
i_{g d}=p_{g 0} / u_{g d} \\
i_{g q}=0
\end{array}\right.
$$

where $p_{g 0}$ is the active power of the grid side inverter and $u_{g d}$ is the amplitude of the grid voltage vector. As both $d$-axes of the grid side and rotor side inverters are aligned to the same grid voltage, the overall DFIG currents in the $d q$-axes $i_{d}$ and $i_{q}$ can be calculated as: 
Table $1 i_{\text {dref }}$ and $i_{\text {qref }}$ under different operating conditions

\begin{tabular}{lll}
\hline Criterions & $i_{\text {dref }}$ & $i_{\text {qref }}$ \\
\hline$V_{p c c}>0.9$ & $i_{\text {dref }}=i_{\text {doref }}$ & $i_{\text {qref }}=0$ \\
$0.2<V_{p c c}<0.9$ and & $i_{\text {dref }}=i_{\text {doref }}$ & $i_{\text {qref }}=a \cdot\left(m-V_{p c c}\right)$ \\
$i_{\text {qref }} \leq \sqrt{I_{\text {max }}^{2}-i_{\text {doref }}^{2}}$ & & \\
$0.2<V_{p c c}<0.9$ and & $i_{\text {dref }}=\sqrt{I_{\text {max }}^{2}-i_{\text {qref }}^{2}}$ & $i_{\text {qref }}=a \cdot\left(m-V_{p c c}\right)$ \\
$i_{\text {qref }}>\sqrt{I_{\text {max }}^{2}-i_{\text {doref }}^{2}}$ & & \\
\hline
\end{tabular}

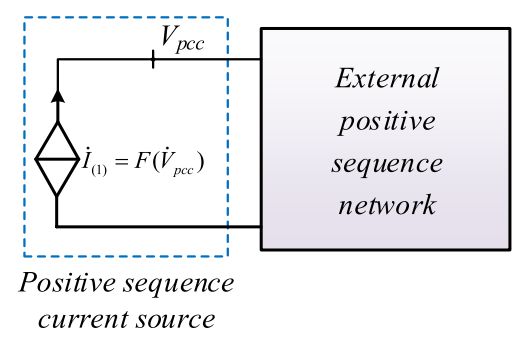

Fig. 2 Positive-sequence equivalent circuit of the DG

$$
\left\{\begin{array}{l}
i_{d}=i_{s d}+i_{g d} \\
i_{q}=i_{s q}
\end{array}\right.
$$

\subsection{Equivalent model of multi-type DGs}

Based on the above analysis, IIDG and DFIG can both be equivalent to a constant positive sequence current source with its amplitude and phase having a functional relationship with the positive sequence voltage at the $P C C$. Thus, the equivalent model can be expressed as $\dot{I}_{D G}=I \angle \varphi_{i u}$ with the magnitude $I$ and the angle difference between the positive sequence current and voltage $\phi_{\text {iи }}$ given as:

$$
\left\{\begin{array}{l}
I=\sqrt{i_{\text {dref }}^{2}+i_{\text {qref }}^{2}} \\
\varphi_{\text {iu }}=\arctan \left(i_{\text {qref }} / i_{\text {dref }}\right)
\end{array}\right.
$$

where $i_{d r e f}$ and $i_{\text {qref }}$ are presented in Table 1

In Table 1, $\alpha$ and $m$ represent the reactive current coefficient and voltage limit specified by the grid integration requirements. $I_{\max }$ is the maximum inverter current limit, which is $120 \%$ of the rated value for IIDG and $L_{m} \cdot I_{\text {rmax }} / L_{s}$ for DFIG. According to (18), an equivalent model of DG can be established, as illustrated in Fig. 2.

\section{PSO-based fast-iterative calculation method of fault components for distribution networks}

Based on the analysis in Sect. 2, a DG can be equivalent to a positive sequence current source under fault conditions. However, the amplitude and phase have a complex nonlinear relationship with the positive sequence voltage at the $P C C$. This results in difficulties in determining the fault components with conventional calculation methods. For instance, in the conventional calculation method with separation of the real and imaginary components, the nonlinear equations can be solved but with low calculation efficiency. In addition, the nonlinearity increases with the increased complexity of the system, which may result in the conventional approach being unable to produce the results within limited time. In this paper, a fastiterative calculation method based on the improved PSO algorithm is proposed to make the nonlinear calculations faster with higher accuracy.

\subsection{Improved PSO algorithm}

PSO is an intelligent algorithm that imitates birds in search of food with the merits of fast convergence, small number of parameter settings and easy implementation [28]. However, PSO is prone to be trapped in local optima, i.e. local convergence. The improvement of the original PSO algorithm was first proposed in [29], and in this paper an adaptive weight coefficient method is applied to avoid local convergence.

The standard PSO algorithm corrects individual behaviors to obtain the optimal solution through information sharing and individual experience among groups. The particle swarm consists of $n$ particles flying in a D-dimensional space with a certain speed. The position of the ith particle is denoted as $X_{i}=\left[x_{i 1}, x_{i 2}, \ldots, x_{i \mathrm{D}}\right]$ and the corresponding velocity is denoted as $V_{i}=\left[v_{i 1}, v_{i 2}, \ldots\right.$, $\left.v_{i \mathrm{D}}\right] . P_{i}=\left[p_{i 1}, p_{i 2}, \ldots, p_{i \mathrm{D}}\right]$ is an optimal position searched by the particle, while $P_{g}=\left[p_{g 1}, p_{g 2}, \ldots, p_{g \mathrm{D}}\right]$ is the aggregation of the optimal positions currently searched by the particle swarm. The $d t h$ velocity $v_{i d}^{k+1}$ and the position $x_{i d}^{k+1}$ at the $(k+1)$ th iteration are:

$$
\left\{\begin{array}{l}
v_{i d}^{k+1}=\omega v_{i d}^{k}+c_{1} r_{1}\left(p_{i d}^{k}-x_{i d}^{k}\right)+c_{2} r_{2}\left(p_{g d}^{k}-x_{i d}^{k}\right) \\
x_{i d}^{k+1}=x_{i d}^{k}+v_{i d}^{k+1}
\end{array}\right.
$$

where $c_{1}$ and $c_{2}$ represent the acceleration constants, and $\omega$ is the inertia weight. $r_{1}$ and $r_{2}$ are random numbers between 0 and 1 . The inertia weight $\omega$ affects the searching capability of the particle. When $\omega$ is small/large, the algorithm has a strong local/global search capability. The weight coefficient $\omega_{i}$ is applied to accelerate the convergence and prevent a local optimal solution, i.e.:

$$
\omega_{i}=\omega_{\max }-\left(\omega_{\max }-\omega_{\min }\right) \frac{N_{i}}{N_{\max }}
$$

where $\omega_{\max }$ represents the initial inertia weight, and $\omega_{\min }$ represents the inertia weight of iteration reaching the maximum number. $N_{i}$ and $N_{\text {max }}$ represent the current and maximum numbers of iterations, respectively. In the 
calculation approach of the traditional distribution network without DGs under faults, predominantly the symmetrical component method is applied. In a distribution network with DGs, each DG can be replaced by a controlled positive sequence current source, though the nonlinear relationship between the current and voltage at the $P C C$ makes it difficult to calculate the fault components. The nonlinear mathematical model can be expressed as:

$$
f(X)=0
$$

where $X=\left[x_{1}, x_{2}, \ldots, x_{n}\right]^{T}$ is a $n \times 1$ vector, consisting of $n$ unknown variables. For the distribution network with DGs, $X$ represents the required fault components and $f(X)=\left[f_{1(x)}, f_{2(x)}, \ldots, f_{m(x)}\right]^{T}$ is an $m$-dimensional vector function. In order to solve (21), the unconstrained optimization approach is applied by converting it into a nonlinear least squares form as:

$$
\min _{X \in R^{n}} \sum_{i=1}^{m} f_{i}^{2}(X)=0
$$

(22) can be transformed into a 2-norn in the vector space as:

$$
\min _{X \in R^{n}}\|f(X)\|_{2}
$$

(23) can then be converted into an unconstrained minimax optimization model, described as:

$$
\min _{X \in R^{n}} \max _{1 \leq i \leq m}\left\{\left|f_{i}(x)\right|\right\}
$$

where $f_{i}(X)$ represents the objective function. (24) can be used as the fitness function which can be solved by the improved PSO algorithm. The fitness of the particle is inversely correlated to the function value.

\subsection{Fast-iterative calculation method}

A symmetrical component method can be used to establish the fitness function for the fault calculation. The fault components can then be determined with the improved PSO algorithm. The specific procedures of the calculation are explained below.

\subsubsection{Establish the equivalent sequence circuit}

To establish the equivalent sequence circuit, the DGs are replaced by positive sequence current sources, while the conventional synchronous generators are equivalent to the voltage sources in series with the reactance. For the negative and zero sequence networks, the DGs are considered as in an open state.

\subsubsection{Establish the voltage equation at each node}

According to each sequence network, there are:

$$
\left\{\begin{array}{l}
\dot{U}_{i(1)}=Z_{i f(1)} \dot{I}_{f(1)}+Z_{D G(1)} \dot{I}_{D G(1)} \\
\dot{U}_{i(2)}=Z_{f(2)} \dot{I}_{f(2)} \\
\dot{U}_{i(0)}=Z_{f(0)} I_{f(0)}
\end{array}\right.
$$

where subscripts (1), (2) and (0) represent the positive, negative and zero sequence networks, respectively. $V_{i}, I_{i}$ and $Z_{i}$ are respectively the voltage, current and impedance of node $i . Z_{\mathrm{DG}}$ is the impedance of the transformer and the DG branch, while $I_{D G}$ is the positive sequence current of the DG.

\subsubsection{Establish constraints of the fault boundary}

The constraints of the fault boundary can be established according to the fault type, e.g., for a two-phase short-circuit fault, the fault boundary constraints are:

$$
\left\{\begin{array}{l}
\dot{U}_{f(1)}=\dot{U}_{f(2)} \\
\dot{I}_{f(1)}+\dot{I}_{f(2)}=0
\end{array}\right.
$$

where $U_{f(1)}$ and $U_{f(2)}$ represent the fault boundary constraints in the positive and negative sequence networks, respectively.

\subsubsection{Define the fitness function}

The equivalent model of the DGs, the voltage relationship and the fault boundary constraints as respectively described in (18), (25) and (26) can be combined to establish the objective function $f_{i}(\mathrm{X})$. The fitness function can be constructed according to (24), and all particle fitness values can then be calculated. For the distribution network with the integration of an IIDG and a DFIG as illustrated in Fig. 5, the vector $\mathrm{X}$ has a dimension of 12 and consists of 12 variables, including the voltage/current amplitudes $\left(M a g_{U-I I D G} / M a g_{I-I I D G}\right)$ and the phase angles $\left(P h i_{U-I I D G} / P h i_{I-I I D G}\right)$ of the IIDG, the voltage/current amplitudes ( $\left.M a g_{U-D F I G} / M a g_{I-I D F I G}\right)$ and the phase angles $\left(P h i_{U-D F I G} / P h i_{\text {I-DFIG }}\right)$ of the DFIG, the current amplitude $\left(\mathrm{Mag}_{I s}\right)$ and the phase angle $\left(P h i_{I_{S}}\right)$ of the system, and the current amplitude $\left(\mathrm{Mag}_{I f}\right)$ ) and the phase angle $\left(P h i_{I f}\right)$ at the faulty point. The objective functions are defined in Table 2 under different faults where 3P-SCF, 2P-SC-F, 2P-G-F, and 1P-G-F represent a three-phase short-circuit fault, a two-phase short-circuit fault, a twophase to ground fault, and a single-phase to ground fault, respectively.

\subsubsection{Initialize randomly with the improved PSO}

In setting the maximum number of iterations, the number of variables and the size of the particle swarm, the 
Table 2 Objective function under different fault conditions

\begin{tabular}{|c|c|c|c|}
\hline & Equivalent model of the DGs & Voltage relationship & Fault boundary constraints \\
\hline 3P-SC-F & $\int$ Mag $\mid-\| D G=\sqrt{i_{\| D G d r e f}^{2}+i_{\| D G g r e f}^{2}}$ & $\dot{E}_{S}=\dot{i}_{s} Z_{s}-\dot{U}_{I I D G}+\dot{l}_{I D G} Z_{L T 1}$ & $\dot{U}_{f(2)}=0$ \\
\hline 2P-SC-F & $\left\{P h i_{I-\| D G}=\arctan \left(\frac{i_{I I D G d a r e f}}{i_{I I D G q u e f}}\right)\right.$ & $\begin{array}{l}\dot{U}_{I D G}-\dot{I}_{I D G} Z_{L T 1}=\dot{U}_{D F I G}-\dot{I}_{D F I G} Z_{L T 2} \\
\dot{U}_{I I D G}-\dot{I}_{I I D G} Z_{L T 1}=\left(\dot{i}_{s}+\dot{I}_{I D G}+\dot{I}_{D F I G}\right) Z_{L 2}-\dot{U}_{f(1)} \\
\dot{U}_{f(1)}=\left(\dot{l}_{f(1)}+\dot{I}_{S}+\dot{I}_{I D G}+\dot{I}_{D F I G}\right) Z_{L D 3}\end{array}$ & $\left\{\begin{array}{l}\dot{U}_{f(1)}=\dot{U}_{f(2)} \\
\dot{l}_{f(1)}+\dot{I}_{f(2)}=0\end{array}\right.$ \\
\hline $2 \mathrm{P}-\mathrm{G}-\mathrm{F}$ & $\int M_{\text {Mag }-D F I G}=\sqrt{i_{D F I G d r e f}^{2}+i_{D F / G q r e f}^{2}}$ & $\dot{U}_{f(2)}=\frac{\left(Z_{S(2)}+Z_{L 2(2)}\right) Z_{L D 3(2)}}{Z_{S(2)}+Z_{L 2(2)}+Z_{L D 3(2)}} \dot{l}_{f(2)}$ & $\left\{\begin{array}{l}\dot{U}_{f(1)}=\dot{U}_{f(2)} \\
\dot{l}_{f(1)}+\dot{I}_{f(2)}=0\end{array}\right.$ \\
\hline 1P-G-F & $P h i_{1-D F I G}=\arctan \left(\frac{i_{D F / G d r e f}}{i_{\text {DFIGaref }}}\right)$ & & $\left\{\begin{array}{l}\dot{U}_{f(1)}=\dot{U}_{f(2)}=0 \\
\dot{I}_{f(1)}+\dot{I}_{f(2)}\end{array}\right.$ \\
\hline
\end{tabular}

speed and position of the particles are randomly initialized in the particle swarm. The constraints of the initial position are:

$$
\left\{\begin{array}{l}
\operatorname{mag}\left(\dot{U}_{i(1)}\right)>0 \\
-\pi<\operatorname{angle}\left(\dot{U}_{i(1)}\right)<\pi
\end{array}\right.
$$

\subsubsection{Update the global optimal solution}

The extremum of each individual is the optimal solution obtained for each particle and a global value is identified from these optimal solutions as the global optimal solution for this iteration, which is then compared with the historical global optimization to update its value.

\subsubsection{Update inertia weight, speed and position}

The inertia weight is calculated with (20), while the velocity and position of the particle are updated and bounded according to (19).

\subsubsection{Evaluate iteration}

It is evaluated whether the precision $e$ or maximum number of iterations is achieved. If the requirement is met, the method continues to Step 9, otherwise, it goes back to Step 6.

\subsubsection{Calculate the fault components}

The fault current components of each branch are calculated based on the calculated node voltage and network impedance according to (25).

A comprehensive flow chart of the proposed fast-iterative calculation method is depicted in Fig. 3.

\section{Simulation system and case studies}

\subsection{Simulation system}

The distribution network with an IIDG and a DFIG is established in PSCAD/EMTDC, as shown in Fig. 4. Two different fault conditions at point $f$, i.e., a three-phase short-circuit fault and a two-phase short-circuit fault, are simulated. The proposed fast-iterative calculation method is used to calculate the fault components from the established equivalent model under symmetrical and asymmetrical faults. The calculation results are compared with those of the simulations for verification. The

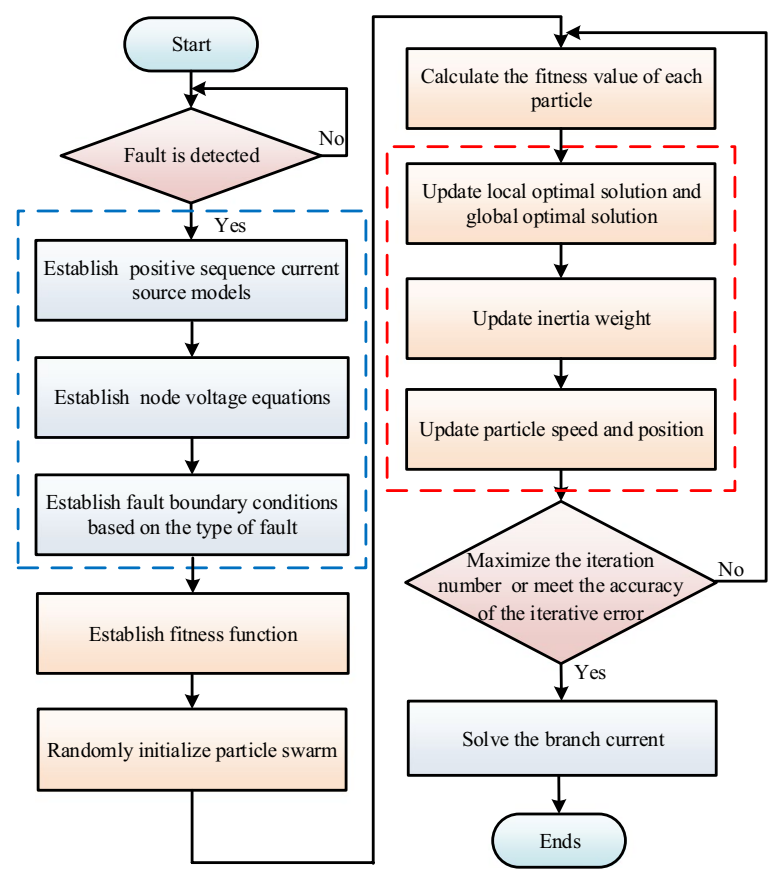

Fig. 3 Flow chart of the proposed fast-iterative calculation method

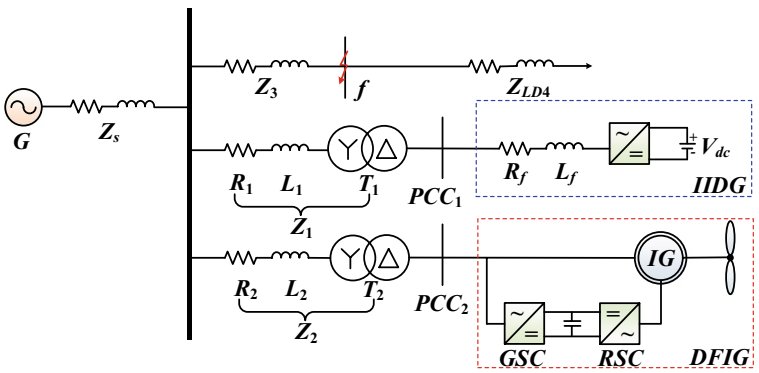

Fig. 4 Equivalent model of the distribution network with multi-type DGs 
main parameters of the simulation system are listed in Table $3[15,22,30]$.

\subsection{Case studies}

\subsubsection{Symmetrical fault}

When a three-phase fault occurs at point $f$ at $1 \mathrm{~s}$, the $P C C$ voltages of the IIDG and DFIG are presented in Figs. 5 and 6, respectively. When the fault occurs, the voltage of the IIDG drops to 0.295 p.u. and the voltage of the DFIG drops to 0.365 p.u. within $0.03 \mathrm{~s}$.

The fault current characteristics of the IIDG and DFIG are presented in Figs. 7 and 8, respectively. The current references of the IIDG and DFIG are calculated based on the respective $P C C$ voltages. From (1), (2) and (14), $i_{\text {dref }}$ and $i_{\text {qref }}$ of the IIDG are 1 p.u. and 0 p.u. before the fault, while $i_{\text {rdref }}$ and $i_{\text {rqref }}$ of the DFIG are 1.107 p.u. and $-0.44 \mathrm{p} . u$. before the fault. When the fault occurs, the $d$-axis current of the IIDG tracks the reference ( 0 p.u.) within $0.01 \mathrm{~s}$ and the $q$-axis current tracks the reference (1.2 p.u.) within $0.05 \mathrm{~s}$ as shown in Fig. 7. In Fig. 8, the $d$-axis current of the DFIG tracks the reference $(0.977$ p.u.) within $0.02 \mathrm{~s}$ and the $q$-axis current tracks the reference $(0.698$ p.u.) within $0.04 \mathrm{~s}$. The maximum overshoot is $13.24 \%$ and the average tracking error after reaching the steady state is approximately $2.4 \%$. This indicates that

Table 3 Main parameters of the simulation system

\begin{tabular}{ll}
\hline Parameter & Value \\
\hline Rated capacity $S_{N} / \mathrm{MVA}$ & 10 \\
Rated AC voltage $U_{N} / \mathrm{kV}$ & 10.5 \\
System equivalent impedance $Z_{S} / \Omega$ & $j \cdot 1$ \\
Impedance $Z_{1} / \Omega$ & $0.85+j \cdot 2.5$ \\
Impedance $Z_{2} / \Omega$ & $0.85+j \cdot 2.5$ \\
Impedance $Z_{3} / \Omega$ & $0.85+j \cdot 1.97$ \\
Impedance $Z_{L D 4} / \Omega$ & $10+j 9.42$ \\
IIDG rated capacity $S_{\text {IIDG }} / \mathrm{MVA}$ & 10 \\
IIDG rated voltage $U_{\text {IIDG }} / \mathrm{kV}$ & 0.38 \\
DFIG rated capacity $S_{D F I G} / \mathrm{MVA}$ & 10 \\
DFIG rated voltage $U_{D F I G} / \mathrm{kV}$ & 0.69 \\
Stator winding resistance $R_{r} / \Omega$ & 1.096 \\
Stator leakage reactance $L_{\text {so }} / \mathrm{H}$ & 0.066 \\
Rotor winding resistance $R_{r} / \Omega$ & 0.772 \\
Rotor leakage reactance $L_{r d} / H$ & 0.066 \\
Mutual reactance $L_{m} / H$ & 1.004 \\
Rated speed $\omega_{r} / r p m$ & 1800 \\
Maximum number of iterations & 500 \\
Population size & 500 \\
Particle coefficients $C_{1} / C_{2}$ & $2 / 2$ \\
Limiting values of the inertia coefficient $\omega_{\text {max }} / \omega_{\text {min }}$ & $0.9 / 0.4$ \\
Accuracy of the iterative error $e$ & $1 \mathrm{e}-6$ \\
\hline
\end{tabular}

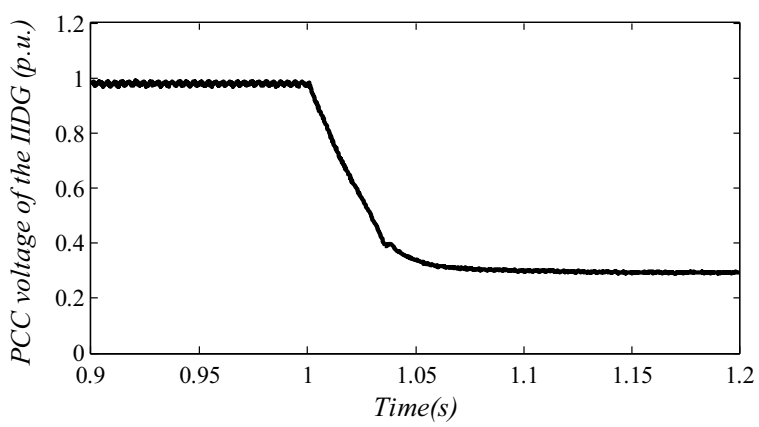

Fig. 5 PCC voltage of the IIDG

the output currents can rapidly track the references and conform to the LVRT strategies.

The theoretical fault current calculations are obtained by the proposed fast-iterative calculation method, and are compared to the simulation results in Table 4, while the percentage magnitude and phase errors between the theoretical calculations and simulation results are depicted in Fig. 9. These indicate that the results are very close and the maximum error is within $2 \%$. This demonstrates the effectiveness of the equivalent model of the distribution network with multi-type DGs and the proposed fast-iterative calculation method with high accuracy under a symmetrical fault.

\subsubsection{Asymmetrical fault}

When a two-phase short-circuit fault occurs at point $f$, the theoretical calculations and simulation results are compared in Table 5. As can be seen, the negative sequence currents of the IIDG and DFIG are mitigated to $0.038 \mathrm{kA}$ and $0.042 \mathrm{kA}$ respectively by the controls. As both negative sequence currents are sufficiently small, the IIDG and DFIG can be equivalent to positive sequence current sources under the asymmetrical fault. As shown in Table 5, the calculation and simulation results of the amplitude and phase of the currents are very close. In addition, the percentage magnitude and phase errors between the theoretical calculations and

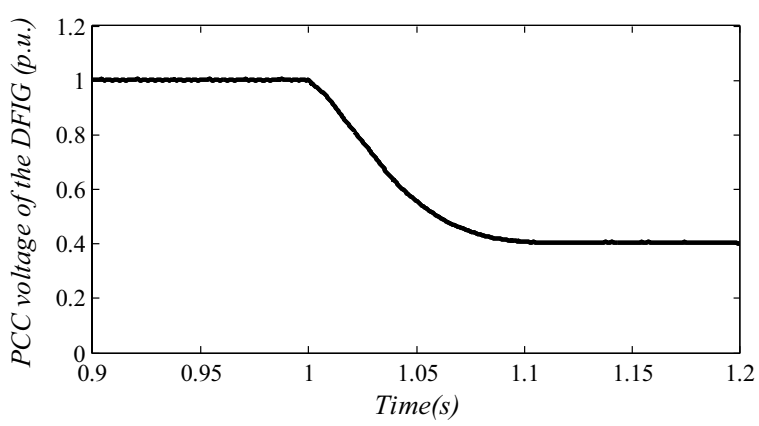

Fig. 6 PCC voltage of the DFIG 


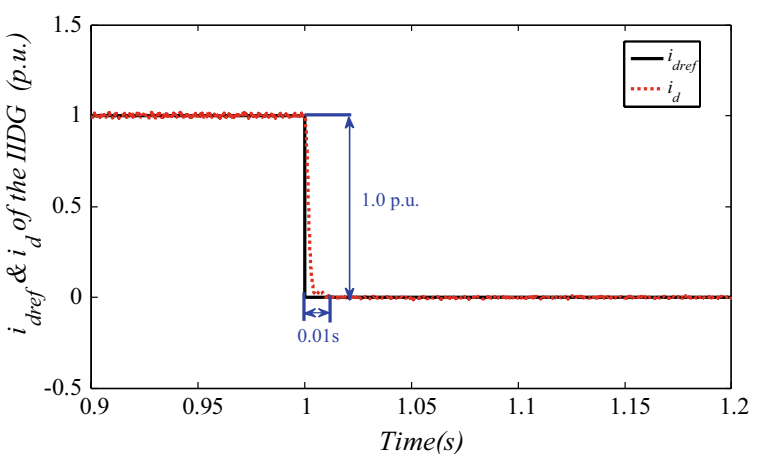

(a) IIDG $d$-axis current

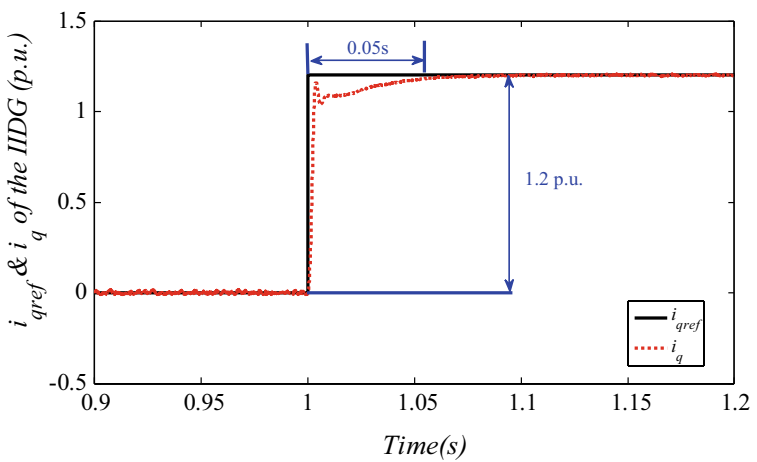

(b) IIDG $q$-axis current

Fig. 7 Fault current characteristics of the IIDG under a three-phase fault. a IIDG $d$-axis current. b IIDG q-axis current

simulation results as shown in Fig. 10 indicate a maximum error of less than $1 \%$, validating the effectiveness of the equivalent model and the proposed fast-iterative calculation method with high accuracy under an asymmetrical fault.

The convergence of the fast-iterative calculation method is evaluated under a three-phase short-circuit fault as an example, and the error precision of the algorithm is presented in Fig. 11. When the number of iterations increases, the error precision of the algorithm decreases, which demonstrates the convergence performance of the proposed fast-iterative calculation method. When the iteration reaches 284 times, the error precision reaches the set value $e$. The improved PSO algorithm exits the loop and the correct fault components are produced. The proposed fast-iterative calculation method is compared with the conventional approach with the separation of real and imaginary components in Table 6 [15, $22,31]$. As can be seen there, the fault current calculation results of the two approaches are very close.

The comparison demonstrates that the proposed calculation method of the fault components has high accuracy and can assist with the settings of the protection threshold. Using a computer with an Intel Core

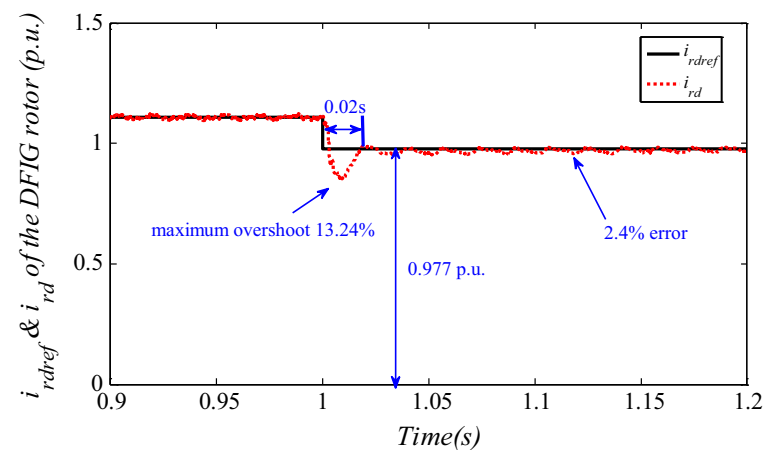

(a) DFIG rotor $d$-axis current

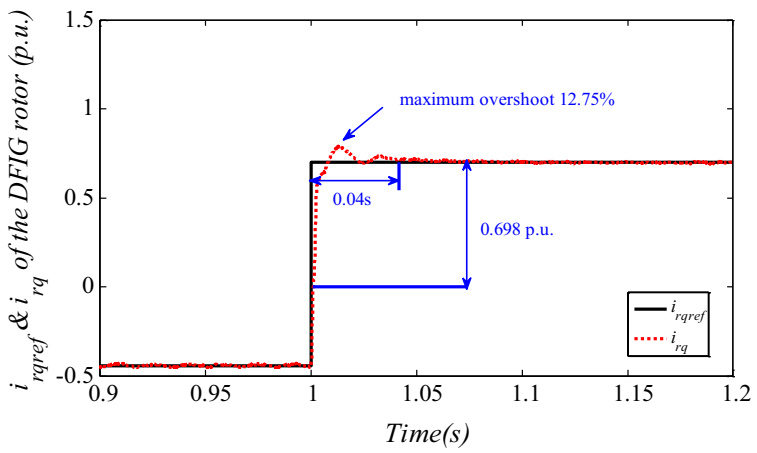

(b) DFIG rotor $q$-axis current

Fig. 8 Fault current characteristics of the DFIG rotor under a three-phase fault. a DFIG rotor $d$-axis current. $\mathbf{b}$ DFIG rotor $q$-axis current

i7-6700HQ processor and 16 GB RAM, the calculation time of the improved PSO algorithm is $2.75 \mathrm{~s}$, whereas the calculation time of the traditional algorithm is $42.48 \mathrm{~s}$, which is over 15 times of that of the proposed approach. Hence, the calculation method proposed in this paper is fast and has high efficiency, and can meet the requirements in practical application.

\section{Conclusions}

In this paper, a distribution network with multi-type DGs has been investigated, and the fault current characteristics of the IIDG and DFIG have been analyzed. Based on an analytical study, an equivalent model of multi-type DGs has been established. A fast-iterative calculation method for fault current component calculation, which has high calculation efficiency while avoids local convergence, has been proposed. Specifically, the following conclusions are drawn.

1. Fault current characteristics and analysis: Based on the LVRT strategy, the IIDG only outputs positive sequence current under both symmetrical and asym- 
Table 4 Fault current calculations/simulations under a three-phase fault at point $f$

\begin{tabular}{|c|c|c|c|c|c|c|c|c|}
\hline & Current fr & network-side & Current o & & Current o & & Current a & It point \\
\hline & $\mathrm{Amp} / \mathrm{kA}$ & Phase $/^{\circ}$ & Amp/kA & Phase $/^{\circ}$ & $\mathrm{Amp} / \mathrm{kA}$ & Phase/ ${ }^{\circ}$ & $\mathrm{Amp} / \mathrm{kA}$ & Phase/ ${ }^{\circ}$ \\
\hline Theoretical calculations & 2.769 & -84.27 & 0.662 & 94.49 & 0.676 & 67.61 & 1.546 & 108.10 \\
\hline Simulation results & 2.782 & -84.48 & 0.66 & 95.41 & 0.671 & 66.45 & 1.54 & 108.77 \\
\hline
\end{tabular}

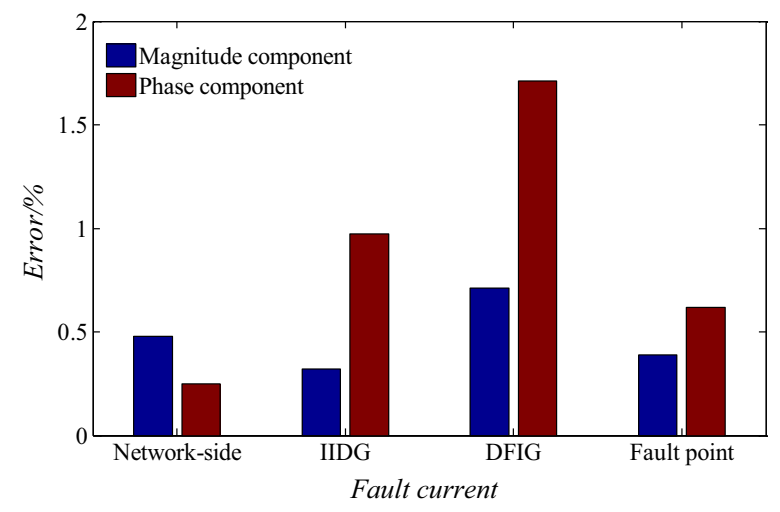

Fig. 9 Error comparison of current calculations under a symmetrical fault metrical faults. The fault current of the DFIG has been derived with consideration of the control strategy for balancing the stator current.

2. Equivalent model of DGs under faults: The DGs can be equivalent to a positive sequence current source without negative and zero sequence current. The amplitude and phase of the current source have a functional relationship with the positive sequence voltage at the PCC.

3. Fast-iterative calculation method: The traditional algorithm which extracts the real and imaginary components from the nonlinear equations to form the linear equations for calculating the fault components is time-consuming and not appropriate for a distribution network with DGs. A fast-iterative cal-

Table 5 Fault current calculations/simulations under a two-phase fault at point $f$

\begin{tabular}{|c|c|c|c|c|c|c|c|c|}
\hline & \multicolumn{4}{|c|}{ Current from network-side } & \multicolumn{4}{|c|}{ Current of IIDG } \\
\hline & \multicolumn{2}{|c|}{ Positive sequence } & \multicolumn{2}{|c|}{ Negative sequence } & \multicolumn{2}{|c|}{ Positive sequence } & \multicolumn{2}{|c|}{ Negative sequence } \\
\hline & Amp/kA & Phase $^{\circ}$ & Amp/kA & Phase $^{\circ}$ & Amp/kA & Phase/ ${ }^{\circ}$ & Amp/kA & Phase $^{\circ}$ \\
\hline Theoretical calculations & 1.9629 & 266.42 & 0.6715 & 107.65 & 0.658 & 74.30 & 0 & / \\
\hline \multirow[t]{4}{*}{ Simulation results } & 1.9658 & 265.25 & 0.6732 & 108.48 & 0.66 & 74.83 & 0.038 & -76.16 \\
\hline & \multicolumn{4}{|c|}{ Current of DFIG } & \multicolumn{4}{|c|}{ Current at fault point } \\
\hline & \multicolumn{2}{|c|}{ Positive sequence } & \multicolumn{2}{|c|}{ Negative sequence } & \multicolumn{2}{|c|}{ Positive sequence } & \multicolumn{2}{|c|}{ Negative sequence } \\
\hline & Amp/kA & Phase $/^{\circ}$ & Amp/kA & $\mathrm{Phase}^{\circ}$ & Amp/kA & Phase $/^{\circ}$ & Amp/kA & Phase $^{\circ}$ \\
\hline Theoretical calculations & 0.647 & 47.33 & 0 & / & 0.805 & 110.15 & 0.805 & 110.15 \\
\hline Simulation results & 0.6424 & 47.01 & 0.042 & 72.73 & 0.806 & 112.68 & 0.806 & 112.95 \\
\hline
\end{tabular}

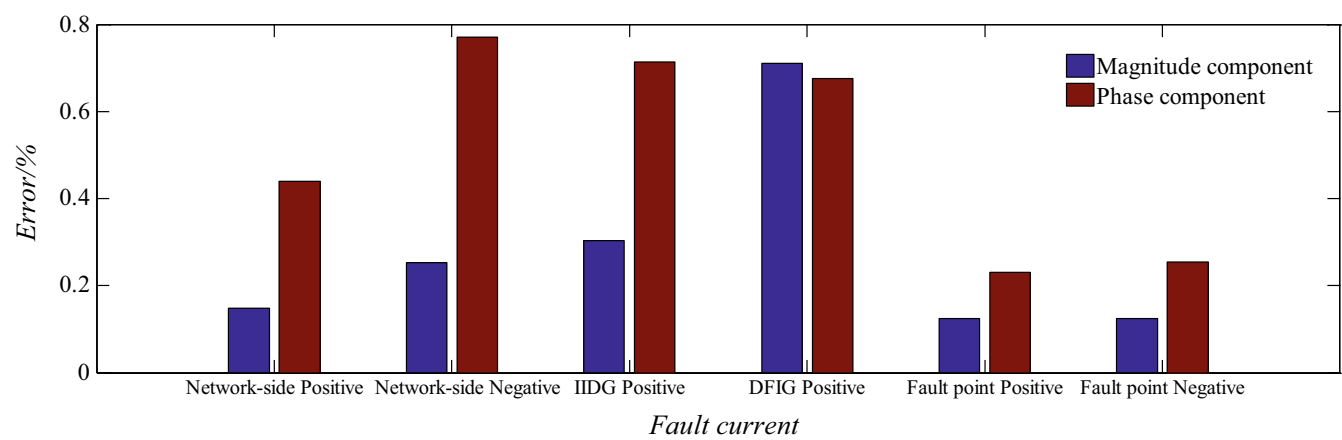

Fig. 10 Error comparison of current calculations/simulations under an asymmetrical fault 
Table 6 Comparison of the fast-iterative calculation method with the conventional algorithm under a three-phase fault at point $f$

\begin{tabular}{|c|c|c|c|c|c|c|c|c|}
\hline & \multicolumn{2}{|c|}{ Current from network-side } & \multicolumn{2}{|c|}{ Current of IIDG } & \multicolumn{2}{|c|}{ Current of DFIG } & \multicolumn{2}{|c|}{ Current at fault point } \\
\hline & Amp/kA & Phase $/^{\circ}$ & $\mathrm{Amp} / \mathrm{kA}$ & Phase $/^{\circ}$ & Amp/kA & Phase $/^{\circ}$ & Amp/kA & Phase $/^{\circ}$ \\
\hline $\begin{array}{l}\text { Fast-iterative cal- } \\
\text { culation method }\end{array}$ & 2.769 & -84.27 & 0.662 & 94.49 & 0.6758 & 67.61 & 1.546 & 108.10 \\
\hline $\begin{array}{l}\text { Traditional algo- } \\
\text { rithm }\end{array}$ & 2.755 & -84.28 & 0.66 & 94.96 & 0.673 & 65.596 & 1.55 & 108.6 \\
\hline
\end{tabular}

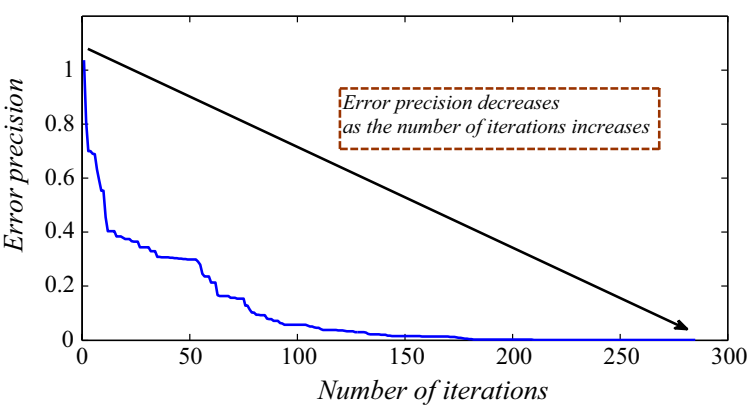

Fig. 11 Trend of error of the fast-iterative calculation method

culation method based on an improved PSO algorithm has been proposed to achieve global optimization and enhance efficiency. Specific procedures of the method have been explained.

4. Verification and error precision: The proposed equivalent model and the faster-iterative calculation method have been simulated and verified in PSCAD/ EMTDC. The simulation results have validated the effectiveness of the equivalent model and the proposed calculation method. These can meet the calculation and protection setting requirements. In addition, an error precision for fast-iterative calculation has been provided.

It is noted that the PSO algorithm may not be the optimal algorithm and is unlikely to be the only approach that can solve the nonlinear equations presented in this paper. Thus, further investigation will be carried out in future research.

\section{Acknowledgements}

Not applicable.

\section{Authors' contributions}

The paper was a collaborative effort among the authors. PW conceived the idea and provided technical guidance required for completing the study. JS formulated the algorithms, carried out the simulation studies, and wrote the paper. FL carried out simulation studies and improved the paper quality. FS provided technical suggestions and guidance. XK provided the technical guidance required for completing the study. GX improved the paper quality. XPZ provided critical comments and edited the manuscript. XG reviewed the manuscript. The author(s) read and approved the final manuscript.

\section{Funding}

This work was supported by National Natural Science Foundation of China under Grant 51807091, the China Postdoctoral Science Foundation under Grant 2019M661846, Open Research Fund of Key Laboratory of Power System Intelligent Dispatch and Control of Ministry of Education, EPSRC under Grant EP/N032888/1, and the International Science and Technology Collaborative Project of Policy Guidance Plan of Jiangsu Province under Grant BZ2018026.

\section{Declarations}

\section{Competing interests}

The authors declare that they have no known competing financial interests or personal relationships that could have appeared to influence the work reported in this paper.

\section{Author details}

${ }^{1}$ School of Automation, Nanjing University of Science \& Technology (NUST), Nanjing 210094, Jiangsu, China. ${ }^{2}$ Key Laboratory of Power System Intelligent Dispatch and Control of Ministry of Education, Shandong University, Jinan 250061, China. ${ }^{3}$ Jiangsu Electric Power Research Institute, Jiangsu Electric Power Company, Nanjing 211103, Jiangsu, China. ${ }^{4}$ School of Engineering, University of Birmingham, Edgbaston B15 2TT, Birmingham, UK. ${ }^{5}$ Nanjing GWDR Relays Technologies Co., Nanjing 210032, Jiangsu, China.

Received: 30 March 2021 Accepted: 19 August 2021

Published online: 07 September 2021

\section{References}

1. Xiao, Y., Ouyang, J., \& Xiong, X. (2020). Fault protection method of singlephase break for distribution network considering the influence of neutral grounding modes. Protection and Control of Modern Power Systems, 5(10), 1322-1332.

2. Li, R., Wong, P., Wang, K., Li, B., \& Yuan, F. (2020). Power quality enhancement and engineering application with high permeability distributed photovoltaic access to low-voltage distribution networks in Australia. Protection and Control of Modern Power Systems, 5(18), 777-786.

3. Konstantelos, I., Giannelos, S., \& Strbac, G. (2017). Strategic valuation of smart grid technology options in distribution networks. IEEE Transactions on Power Systems, 32(2), 1293-1303.

4. Adamo, C., Jupe, S., \& Abbey, C. (2009). Global survey on planning and operation of active distribution networks - Update of CIGRE C6.11 working group activities. In 20th international conference and exhibition on electricity distribution - Part 1 (pp. 1-4).

5. Bian, X., Wang, B., Chen, J., \& Yang, L. (2016). Improvement of low voltage ride through capability of wind farm using coordinated control of the improved DFIG and VSC-HVDC. Power System Protection and Control, 457(1), 17-24.

6. Rashid, G., \& Ali, M. (2017). Nonlinear control-based modified BFCL for LVRT capacity enhancement of DFIG-based wind farm. IEEE Transactions on Energy Conversion, 32(1), 284-295.

7. Mohamed, S., Aruna, P., Devaraj, D., \& Bouzguenda, M. (2019). Performance comparison of active and passive LVRT strategies for grid connected PV systems. In IEEE international conference on intelligent techniques in control, optimization and signal processing (pp. 1-5). 
8. Yang, Z., Wang, L., \& Zhou, H. (2016). The novel fault analysis method of the power grid with inverter interfaced distribution generators. Distributed Generation \& Alternative Energy Journal, 31(2), 55-73.

9. El-Naggar, A., \& Erlich, I. (2016). Analysis of fault current contribution of Doubly-Fed Induction Generator Wind Turbines during unbalanced grid faults. Renewable Energy, 91, 137-146.

10. Ling, Y., Cai, X., \& Wang, N. (2013). Rotor current transient analysis of DFIGbased wind turbines during symmetrical voltage faults. Energy Conversion and Management, 76, 910-917.

11. Pradhan, K., \& Joos, G. (2007). Adaptive distance relay setting for lines connecting wind farms. IEEE Transactions on Energy Conversion, 22(1), 206-213.

12. Wang, Q., Zhou, N., \& Ye, L. (2015). Fault analysis for distribution networks with current-controlled three-phase inverter-interfaced distributed generators. IEEE Transactions on Power Delivery, 30(3), 1532-1542.

13. Bulent, D., Nadar, A., \& Boynuegri, A. A fault analysis method for microgrids consisting of inverter interfaced distributed generators. In 12 th IET international conference on developments in power system protection (pp. $1-5)$.

14. Swain, S., \& Ray, P. (2017). Short circuit fault analysis in a grid connected DFIG based wind energy system with active crowbar protection circuit for ride through capability and power quality improvement. International Journal of Electrical Power \& Energy Systems, 84, 64-75.

15. Kong, X., Zhang, Z., Yin, X., \&Wan, F. (2015). Study of fault current characteristics of DFIG considering impact of crowbar protection. Transactions of China Electrotechnical Society, 30(8), 1-10.

16. Pannell, G., Atkinson, D., \& Zahawi, B. (2010). Analytical study of grid-fault response of wind turbine doubly fed induction generator. IEEE Transactions on Energy Conversion, 25, 1081-1091.

17. Zhou, S., Wang, Q., \& Xiao, L. (2017). Control strategy of low voltage ridethrough for double-fed wind generator with the stator Crowbar circuit mode switch. Power System Protection \& Control, 45(4), 33-39.

18. Li, W., Chao, P., \& Liang, X. (2018). A practical equivalent method for DFIG wind farms. IEEE Transactions on Sustainable Energy, 9(2), 610-620.

19. Boutsika, T., \& Papathanassious, S. (2008). Short-circuit calculations in networks with distributed generation. Electric Power Systems Research, 78(7), 1181-1191.
20. Luo, Z., Su, M., Sun, Y., \& Wang, H. (2017). Stability analysis and concept extension of harmonic decoupling network for the three-phase grid synchronization systems. International Journal of Electrical Power \& Energy Systems, 89, 1-10.

21. State Grid Corporation of China. QB/T 33593-2017. Beijing: China Standard Press, pp. 1-8, 2017.

22. Wang, P., Song, J., Wu, M., Yu, H., Kong, X., \& Zhang, X. -P. (2020). Analysis of fault current characteristics of distribution network with multi-type distributed generations. In IEEE PES general meeting (pp. 1-5).

23. Yang, Z., Wang, L., \& Zhou, H. (2016). The novel fault analysis method of the power grid with inverter interfaced distribution generator. Distributed Generation \& Alternative Energy Journal, 3(2), 55-73.

24. Guo, X., Wu, W., \& Chen, Z. (2011). Multiple-complex coefficient-filterbased phase-locked loop and synchronization technique for three-phase grid-interfaced converters in distributed utility networks. IEEE Transactions on Industrial Electronics, 58(4), 1194-1204.

25. State Grid Corporation of China. GBT-19963-2011. China Standard Press, pp. 1-13, 2011.

26. Zhu, R., Chen, Z., Wu, X., \& Deng, F. (2015). Virtual damping flux-based LVRT control for DFIG-based wind turbine. IEEE Transactions on Energy Conversion, 30(2), 714-725.

27. Xiao, F., Zhang, Z., \& Yin, X. (2015). Fault current characteristics of the DFIG under asymmetrical fault conditions. Energies, 8(10), 10971-10992.

28. Narges, G., Alibakhsh, K., \& Ashkan, T. (2018). Optimizing a hybrid windPV-battery system using GA-PSO and MOPSO for reducing cost and increasing reliability. Energy, 154, 581-591.

29. Shi, Y., \& Eberhart, R. (1998). Parameter selection in particle swarm optimization. Evolutionary Programming VII, 1447, 591-600.

30. Zou, C., \& Xiao, X. (2016). Integrated protection of DFIG-Based wind turbine with a resistive-type SFCL under symmetrical and asymmetrical faults. IEEE Transactions on Applied Superconductivity, 26(7), 1032-1042.

31. Wang, P., Song, J. (2018). Analysis and iterative calculation of a distribution network with inverted-based distributed generations under fault conditions. In 13th IEEE conference on industrial electronics applications, pp. 1606-1616.

\section{Submit your manuscript to a SpringerOpen ${ }^{\circ}$ journal and benefit from:}

- Convenient online submission

- Rigorous peer review

- Open access: articles freely available online

- High visibility within the field

- Retaining the copyright to your article

Submit your next manuscript at $\boldsymbol{\nabla}$ springeropen.com 